\title{
Clinical Characteristics and Outcomes of Patients Hospitalized for COVID-19 in Africa: Early Insights from the Democratic Republic of the Congo
}

Jean B. Nachega, ${ }^{1,2,3 *} †$ Daniel Katuashi Ishoso, ${ }^{4} \dagger$ John Otshudiema Otokoye,${ }^{5} \dagger$ Michel P. Hermans, ${ }^{6}$ Rhoderick Neri Machekano, ${ }^{7}$ Nadia A. Sam-Agudu, ${ }^{8,9,10}$ Christian Bongo-Pasi Nswe, ${ }^{11,12}$ Placide Mbala-Kingebeni, ${ }^{13}$ Joule Ntwan Madinga, ${ }^{5}$ Stéphane Mukendi, ${ }^{22}$ Marie Claire Kolié, ${ }^{5}$ Edith N. Nkwembe, ${ }^{13}$ Gisele M. Mbuyi, ${ }^{14}$ Justus M. Nsio, ${ }^{14}$ Didier Mukeba Tshialala, ${ }^{15}$ Michel Tshiasuma Pipo, ${ }^{11}$ Steve Ahuka-Mundeke, ${ }^{13}$ Jean-Jacques Muyembe-Tamfum, ${ }^{13}$ Lynne Mofenson, ${ }^{16}$ Gerald Smith, ${ }^{17}$ Edward J. Mills, ${ }^{18}$ John W. Mellors, ${ }^{19}$ Alimuddin Zumla, ${ }^{20,21} \ddagger$ Don Jethro Mavungu Landu, ${ }^{11,12} \ddagger$ and Jean-Marie Kayembe ${ }^{22} \ddagger$

${ }^{1}$ Department of Medicine, Centre for Infectious Diseases, Faculty of Medicine and Health Sciences, Stellenbosch University, Cape Town, South Africa; ${ }^{2}$ Department of Epidemiology and International Health, Johns Hopkins Bloomberg School of Public Health, Baltimore, Maryland;

${ }^{3}$ Department of Epidemiology, Infectious Diseases and Microbiology, Center for Global Health, University of Pittsburgh, Pittsburgh, Pennsylvania;

${ }^{4}$ Community Health Department, Kinshasa School of Public Health, University of Kinshasa, Kinshasa, Democratic Republic of the Congo;

${ }^{5}$ Epidemiological Surveillance Team, COVID-19 Response, Health Emergencies Program, World Health Organization, Kinshasa, Democratic Republic of the Congo; ${ }^{6}$ Department of Endocrinology and Nutrition, Cliniques Universitaires St-Luc, Brussels, Belgium; ${ }^{7}$ African Center of Biostatistics Excellence (ACBE), Faculty of Medicine and Health Sciences, Stellenbosch University, Cape Town, South Africa; ${ }^{8}$ Department of Pediatrics, Institute of Human Virology, University of Maryland School of Medicine, Baltimore, Maryland; ${ }^{9}$ International Research Center of Excellence, Institute of Human Virology Nigeria, Abuja, Nigeria; ${ }^{10}$ Department of Paediatrics, University of Cape Coast School of Medical Sciences, Cape Coast, Ghana; ${ }^{11}$ Department of Public Health, Centre Interdisciplinaire de Recherche en Ethnopharmacologie, Faculty of Medicine, Université Notre-Dame du Kasayi, Kananga, Democratic Republic of the Congo; ${ }^{12}$ Faculty of Public Health, Université Moderne de Kinkole, Kinshasa, Democratic Republic of the Congo; ${ }^{13}$ Department of Medical Microbiology and Virology, Faculty of Medicine, University of Kinshasa, National Institute of Biomedical Research (INRB), Kinshasa, Democratic Republic of the Congo; ${ }^{14}$ Direction Surveillance Épidémiologique (DSE), Direction Générale de Lutte contre la Maladie (DGLM), Ministère de la Santé Publique et Riposte COVID-19, Kinshasa, Democratic Republic of the Congo; ${ }^{15}$ Faculty of Medicine, University of Mbuji-Mayi, Mbuji-Mayi, Democratic Republic of the Congo; ${ }^{16}$ Elizabeth Glaser Pediatric AIDS Foundation, Washington, District of Columbia; ${ }^{17}$ Department of Real World \& Advanced Analytics, Cytel, Vancouver, Canada; ${ }^{18}$ Department of Health Research Methods, Evidence, and Impact, McMaster University, Hamilton, Canada; ${ }^{19}$ Division of Infectious Diseases, Department of Medicine, University of Pittsburgh, School of Medicine, Pittsburgh, Pennsylvania; ${ }^{20}$ Division of Infection and Immunity, Centre for Clinical Microbiology, University College London, London, United Kingdom; ${ }^{21}$ National Institute for Health Research Biomedical Research Centre, University College London Hospitals NHS Foundation Trust, London, United Kingdom; ${ }^{22}$ Department of Internal Medicine, School of Medicine, University of Kinshasa, Kinshasa, Democratic Republic of Congo

\begin{abstract}
Little is known about the clinical features and outcomes of SARS-CoV-2 infection in Africa. We conducted a retrospective cohort study of patients hospitalized for COVID-19 between March 10, 2020 and July 31, 2020 at seven hospitals in Kinshasa, Democratic Republic of the Congo (DRC). Outcomes included clinical improvement within 30 days (primary) and in-hospital mortality (secondary). Of 766 confirmed COVID-19 cases, 500 (65.6\%) were male, with a median (interquartile range [IQR]) age of $46(34-58)$ years. One hundred ninety-one (25\%) patients had severe/critical disease requiring admission in the intensive care unit (ICU). Six hundred twenty patients $(80.9 \%)$ improved and were discharged within 30 days of admission. Overall in-hospital mortality was $13.2 \%$ (95\% Cl: 10.9-15.8), and almost $50 \%$ among those in the ICU. Independent risk factors for death were age $<20$ years (adjusted hazard ratio [aHR] $=6.62,95 \% \mathrm{Cl}: 1.85-23.64$ ), 40-59 years $(\mathrm{aHR}=4.45,95 \% \mathrm{Cl}: 1.83-10.79)$, and $\geq 60$ years (aHR $=13.63,95 \% \mathrm{Cl}: 5.70-32.60)$ compared with those aged $20-39$ years, with obesity $(\mathrm{aHR}=2.30,95 \% \mathrm{Cl}: 1.24-4.27)$, and with chronic kidney disease $(\mathrm{aHR}=5.33,95 \% \mathrm{Cl}$ : 1.85-15.35). In marginal structural model analysis, there was no statistically significant difference in odds of clinical improvement (adjusted odds ratio $[\mathrm{aOR}]=1.53,95 \% \mathrm{Cl}$ : $0.88-2.67, P=0.132)$ nor risk of death $(\mathrm{aOR}=0.65,95 \% \mathrm{Cl}$ : $0.35-1.20)$ when comparing the use of chloroquine/azithromycin versus other treatments. In this DRC study, the high mortality among patients aged $<20$ years and with severe/critical disease is of great concern, and requires further research for confirmation and targeted interventions.
\end{abstract}

\section{INTRODUCTION}

SARS-CoV-2 infection and COVID-19 arrived later in subSaharan Africa (SSA) than in most other regions of the world. As of August 26, 2020, there were 1,014,834 cases and 20,787 deaths $(2.1 \%$ case fatality rate [CFR]) in the WHO African Region. ${ }^{1}$ The high numbers of cases and deaths expected in SSA have not been witnessed to date, despite relatively weak health systems and other barriers limiting comprehensive

\footnotetext{
*Address correspondence to Jean B. Nachega, Department of Epidemiology, Infectious Diseases and Microbiology, Center for Global Health, University of Pittsburgh Graduate School of Public Health, 130 DeSoto St., Crabtree Hall A531, Pittsburgh, PA 15261. E-mail: jbn16@pitt.edu

†These authors contributed equally to this work.

$\ddagger$ These authors contributed equally to this work.
}

implementation of public health interventions. ${ }^{2}$ Several explanations have been hypothesized for this unexpected finding, including early lockdowns, low SARS-CoV-2 testing capacity, a younger population, and concomitant crossimmunity from parasitic diseases and other circulating coronaviruses. ${ }^{3-6}$

The Democratic Republic of the Congo (DRC) confirmed its first COVID-19 case on March 10, 2020 and within 2 weeks declared a state of emergency that included travel bans, lockdowns, widespread testing, and quarantine. ${ }^{6}$ As of August 26, 2020, the DRC has reported 9,891 COVID-19 cases and 251 deaths $(2.5 \%$ CFR), with the capital city Kinshasa being the epicenter. With increased testing, more COVID-19 cases are being reported in SSA, ${ }^{3-5}$ but data on sociodemographic/ clinical characteristics and outcomes among hospitalized patients are still scanty. It is important to ascertain whether features of COVID-19 in Africa differ from those in non-African 
countries. ${ }^{7,8}$ Furthermore, in SSA, there are little data on the prevalence of SARS-CoV-2 coinfection or comorbidity with noncommunicable diseases (NCDs) (e.g., hypertension, diabetes, and obesity) and communicable diseases (e.g., HIV, tuberculosis [TB], and malaria), which may influence COVID19 presentations and outcomes. ${ }^{9,10}$ We aimed to describe clinical characteristics, laboratory features, and outcomes of hospitalized patients with COVID-19 in DRC and to differentiate them from other non-African populations.

\section{METHODS}

Study design, study population, and criteria for hospital admission. We conducted a cohort analysis using routinely collected data from the DRC Ministry of Health's COVID-19 Multi-Sectoral Response Committee database, spanning March 10, 2020-July 31, 2020. All COVID-19 patients admitted at the seven largest health facilities in Kinshasa (one private, two faith-based Catholic, and four public) were eligible for inclusion. Patients were staged according to the WHO COVID-19 clinical categories of mild, moderate, severe, and critical disease (Supplemental Table 1). ${ }^{11}$ The decision to hospitalize patients was based on signs or symptoms of moderate/severe disease, comorbidities, pregnancy, or the development of complications in cases initially managed at home.

Predictors and outcomes variables. Using standardized data collection forms, we extracted sociodemographic, clinical (including comorbidities), laboratory, COVID-19 treatment, and current medication data. Outcomes of interest were clinical improvement within 30 days (primary) and in-hospital mortality (secondary).

SARS-CoV-2 RT-PCR testing. Oropharyngeal or nasal samples were processed at the Virology Laboratory of the National Institute for Biomedical Research in Kinshasa. Samples were tested for SARS-CoV-2 RNA by either BGI RTPCR using the ABI 7500 Fast Applied Biosystems instrument (Thermo Fisher Scientific, Waltham, MA) or Xpert Xpress SARS-CoV-2 using the GeneXpert platform (Cepheid, Sunnyvale, CA), following the manufacturers' instructions.

Case management procedures. On admission, a detailed history, physical examination, including pulse oximetry, was performed. Self-reported HIV and TB status was further corroborated on admission with a review and confirmation of documented medical record information on relevant medications and/or care for these coinfections. The same was carried out for patients self-reporting NCD comorbidities (e.g., hypertension and diabetes). Patients were treated with symptom management, supplemental oxygen, and compassionate treatment protocols according to national guidelines in effect at the time. ${ }^{12}$ Mild cases were treated with hydroxychloroquine (HCQ)/chloroquine (CQ) + azithromycin (AZ), and moderate cases were treated with $\mathrm{HCQ} / \mathrm{CQ}+\mathrm{AZ}$ (Option 1) or lopinavir/ritonavir (LPV/r) (Option 2) + enoxaparin (prophylactic low-molecular weight heparin). ${ }^{10}$ Severe cases were treated with $\mathrm{HCQ} / \mathrm{CQ}+\mathrm{AZ}+$ third-generation cephalosporin + enoxaparin and assisted ventilation (Option 1), or remdesivir + third-generation cephalosporin + enoxaparin + vitamin C and assisted ventilation (Option 2), or HCQ/CQ + (LPV/r) + thirdgeneration cephalosporin + enoxaparin + dexamethasone and assisted ventilation (Option 3). ${ }^{12}$ As of August 24, 2020, remdesivir has not yet been licensed in the $\mathrm{DRC}$, and not all patients received all indicated treatments because of lack of availability.

Statistical analysis. We summarized baseline demographic and clinical characteristics using frequencies and proportions by clinical stage at presentation. Continuous variables were summarized using medians (IQR). Chi-square tests were used to compare proportions and Wilcoxon rank-sum tests to compare medians between mild/moderate and severe/critical cases. COVID-19 symptom resolution was assessed by comparing proportions of patients with symptoms at day 1 (day of admission) versus 10 days later, using the chi-square test for marginal homogeneity. Laboratory values were compared at day 1 and day 10 using the Wilcoxon signed-rank test.

We estimated the proportion of patients with clinical improvement, stratified by baseline demographic and clinical characteristics. Factors associated with clinical improvement at $P$-value $<0.1$ in unadjusted univariable logistic regression were included in a multivariable logistic regression model to identify independent factors associated with clinical improvement. The strength of the association was expressed as adjusted odds ratios and accompanying 95\% Cls. Similarly, we estimated the hazard of death stratified by baseline characteristics and identified factors independently associated with death using Cox regression. The final regression model was performed after the proportionality of hazards assumption was confirmed by a nonsignificant global test and Schoenfeld residuals with horizontal tendency, as well as the presence of parallelism in the $-\ln [-\ln (S(t))]$ plot.

We summarized the strength of association between factors and death using adjusted hazard ratios and associated 95\% Cls. We used a marginal structural model (MSM) based on inverse probability of treatment weighting (IPTW) to assess the efficacy of the $C Q+A Z$ combination versus other therapy, with death as the outcome. Gender, age, WHO stage of disease at admission, hypertension, diabetes mellitus, asthma/ chronic obstructive pulmonary disease (COPD), heart disease, chronic kidney disease (CKD), HIV, TB, obesity, and cancer were included in the treatment model as potential confounders. All analyses were performed using Stata software version 16.1 (College Station, TX). The Venn diagram illustrations of comorbidities and their combination were completed in R Studio Version 1.3.959, May 2020 (R Studio Inc., Boston, MA). ${ }^{13}$

Regulatory approvals. The study was approved by the University of Kinshasa School of Public Health's Ethics Committee (NESP/CE/114/2020 - July 17, 2020); the Institutional Review Board of the University of Pittsburgh, PA (STUDY20080174); and the DRC's National COVID-19 MultiSectorial Response Committee and National Institute of Biomedical Research.

\section{RESULTS}

Sociodemographic and clinical characteristics of hospitalized confirmed cases. Of 852 confirmed COVID-19 cases admitted, we analyzed 766 (89.9\%) with complete information (Figure 1). Baseline sociodemographic characteristics and clinical stage did not differ between patients who were excluded and included in the analysis (Supplemental Table 2). Table 1 summarizes patient characteristics at admission by disease severity. The median (IQR) age was 46 (34-58) years, with $23.3 \%$ aged $\geq 60$ years. Thirty-four $(4.5 \%)$ 


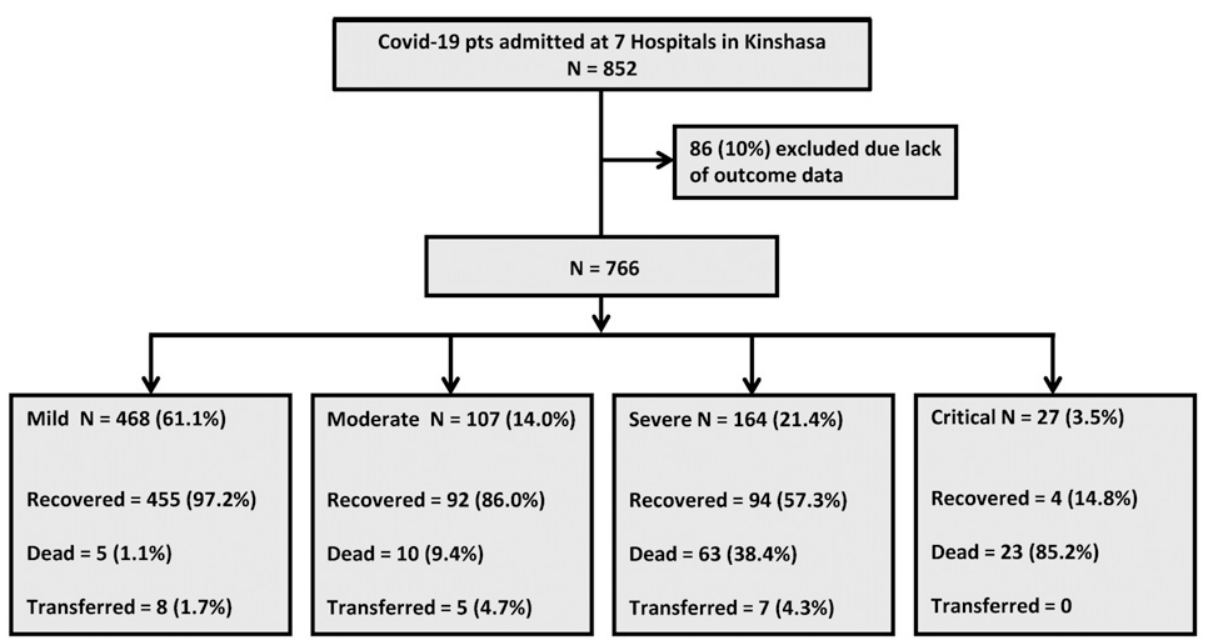

FIGURE 1. Study flow chart.

patients were $<20$ years, with a median age (IQR) of 14.5 (7-18) years and 11 (32\%) younger than 10 years. Five hundred (65.3\%) patients were male. Among the 262 females admitted, 12 (4.6\%) were pregnant. At admission, 468 (61.1\%) patients had mild, 107 (14.0\%) moderate, 164 (21.4\%) severe, and $27(3.5 \%)$ critical disease. All 191 patients with severe or critical disease (25\% of total) were admitted to the intensive care unit (ICU). Among 510 patients with $\mathrm{SpO}_{2}$ measurements, $38.2 \%$ had $\mathrm{SpO}_{2} \leq 90 \%$ on room air. Four of the 34 children presented with severe or critical disease. Compared with those with mild/moderate COVID-19, severe/critical patients had higher median (IQR) C-reactive protein: $60 \mathrm{mg} / \mathrm{dL}$ (48.0-192.0) versus $24.0 \mathrm{mg} / \mathrm{dL}$ (2.6-54.0), respectively $(P=$ $0.010)$, and median (IQR) D-dimer levels $(\mathrm{ng} / \mathrm{mL}): 342.5$ (246.0-443.0) versus $6.9(2.8-100.0)$, respectively $(P=0.011)$.

Among 764 patients with baseline comorbidity information, $264(34.6 \%)$ reported at least one comorbidity, with 128 $(48.5 \%)$ having more than one comorbidity (Figure 2). The most prevalent comorbidities were hypertension (25.4\%) and diabetes (14.0\%). Self-reported prevalence of obesity was $5.1 \%$, heart disease $3.9 \%$, asthma/COPD $3.4 \%$, CKD $0.9 \%$, active TB $2.5 \%$, and HIV 1.6\%. Patients with severe/critical disease were older and had a higher prevalence of hypertension, heart disease, obesity, diabetes, asthma/COPD, and poorer $\mathrm{SpO}_{2}$ levels than those with mild/moderate disease (Table 1). The majority of patients $(n=630,86.8 \%)$ were treated with CQ/AZ. Eighteen patients of 545 (3.1\%) on CQ/AZ versus 1/67 (1.5\%) on other regimens reported at least one side effect $(P=0.70)$, including pruritus, skin rash, gastrointestinal upset, palpitations, or bradycardia. Overall, 620 patients (80.9\%) improved and were discharged within 30 days; $101(13.2 \%)$ died, and $20(2.6 \%)$ were transferred to home care. Median hospital stay among recovered patients was 13 (IQR: 9-17) days. Of the 12 pregnant women, three presented with severe/critical disease and nine had mild/ moderate disease. Five had comorbidities (one hypertension and obesity, one asthma, and three TB). All 12 pregnant women recovered and were discharged in 30 days. Four children (11.8\%) died; they were 16-, 17-, 17-, and 19-yearolds. Three of four had severe/critical disease (severe pneumonia), and one had moderate disease (pneumonia) at admission.
Clinical and respiratory parameters at days 1 and 10 of hospitalization. Supplemental Table 3 presents the changes in markers of infection among patients with assessments at day 1 and day 10 . The proportion reporting headaches $(23.2 \%$ versus $0.6 \%$, respectively, $P<0.001$ ), fever (39.8\% versus $0.3 \%, P<0.001)$, cough (40.9\% versus $1.5 \%, P<0.001)$, sore throat $(12.0 \%$ versus $0.8 \%, P<0.001)$, rhinorrhea $(9.3 \%$ versus $0.1 \%, P<0.001)$, and dyspnea $(37.8 \%$ versus $1.0 \%, P<$ 0.001 ) decreased markedly between day 1 and day 10 . Median oxygen saturation levels significantly improved from $89 \%$ to 98\% over the same time interval.

Factors associated with clinical improvement. The adjusted model for clinical improvement included age-group, the presence of comorbidities (hypertension, heart disease, diabetes, obesity, CKD, and cancer) and treatment received. Table 2 shows factors independently associated with clinical improvement during the observation time. Patients aged 20-39 years $(\mathrm{aOR}=9.40,95 \% \mathrm{Cl}: 4.78-18.52)$ or $40-59$ years $(\mathrm{aOR}=2.64,95 \% \mathrm{Cl}: 1.64-4.26)$ were more likely to improve than patients $\geq 60$ years. Patients with obesity $(\mathrm{aOR}=0.27$, 95\% Cl: 0.12-0.59) were less likely to improve than nonobese patients. Adjusting for treatment with $C Q / A Z$ and age-group, the odds of clinical improvement among patients with severe/ critical COVID-19 was $87 \%$ lower than among that with mild/ moderate disease (aOR $=0.13,95 \% \mathrm{Cl}$ : 0.08-0.20). In MSM analysis, there was no statistically significant difference in odds of clinical improvement $(\mathrm{aOR}=1.53,95 \% \mathrm{Cl}$ : 0.88-2.67, $P=0.132$ ) when comparing the use of $C Q / A Z$ versus other treatment regimens.

Factors associated with in-hospital mortality. Overall, inhospital mortality was $13.2 \%$ (95\% Cl: 10.9-15.8). The median time between admission and death was 4 days (IQR: 2-5). There were no significant gender differences in mortality (13.0\% females versus $13.4 \%$ males). More patients aged $\geq 60$ years $(32.0 \%)$ died, compared with those $<60$ years $(7.5 \%)$ $(P<0.001)$ (Table 3, Figure 3). In-hospital mortality was greater among patients with severe/critical disease than patients with $\mathrm{mild} /$ moderate disease $(45.0 \%$ versus $2.6 \%$, respectively, $P<$ $0.001)$. Patients $<20$ years $(\mathrm{aHR}=6.62,95 \% \mathrm{Cl}: 1.85-23.64)$, $40-59$ years $(\mathrm{aHR}=4.45,95 \% \mathrm{Cl}: 1.83-10.79)$, and $\geq 60$ years (aHR $=13.63,95 \% \mathrm{Cl}: 5.70-32.60)$ had significantly higher hazards of death than those aged 20-39 years. Significantly 
TABLE 1

Demographics and clinical and laboratory characteristics $(N=766)$

\begin{tabular}{|c|c|c|c|c|}
\hline Characteristic & All patients $(n=766)$ & Severe patients (severe and critical) $(n=191)$ & Non-severe patients (mild and moderate) $(n=575)$ & $P$-value \\
\hline Age median (years) (IQR) & $46(34-58)$ & $58(44-66)$ & $42(32-54)$ & $<0.001$ \\
\hline \multicolumn{5}{|l|}{ Age-group (years), $n(\%)$} \\
\hline$<20$ & $34(4.5)$ & $4(2.1)$ & $30(5.2)$ & $<0.001$ \\
\hline $20-39$ & 248 (32.5) & 31 (16.2) & 217 (37.9) & - \\
\hline $40-59$ & $303(39.7)$ & $67(35.1)$ & 236 (41.3) & - \\
\hline$\geq 60$ & $178(23.3)$ & $89(46.6)$ & $89(15.6)$ & - \\
\hline \multicolumn{5}{|l|}{ Gender, $n(\%)$} \\
\hline Male & $500(65.6)$ & $135(71.1)$ & $365(63.8)$ & 0.078 \\
\hline Female & $262(34.4)$ & 55 (28.9) & 207 (36.2) & - \\
\hline Missing & 4 & 1 & 3 & - \\
\hline \multicolumn{5}{|l|}{ Hypertension, $n(\%)$} \\
\hline Yes & $194(25.4)$ & $87(45.6)$ & $107(18.7)$ & $<0.001$ \\
\hline No & 570 (74.6) & $104(54.4)$ & 466 (81.3) & - \\
\hline Missing & 2 & 0 & 2 & - \\
\hline \multicolumn{5}{|l|}{ Heart disease, $n(\%)$} \\
\hline Yes & $30(3.9)$ & $21(11.1)$ & $9(1.6)$ & $<0.001$ \\
\hline No & $733(96.1)$ & 169 (88.9) & $564(98.4)$ & - \\
\hline Missing & 3 & 1 & 2 & - \\
\hline \multicolumn{5}{|l|}{ Obesity, $n(\%)$} \\
\hline Yes & $39(5.1)$ & $22(11.5)$ & $17(3.0)$ & $<0.001$ \\
\hline No & 725 (94.9) & $169(88.5)$ & $556(97.0)$ & - \\
\hline Missing & 2 & 0 & 2 & - \\
\hline \multicolumn{5}{|l|}{ Diabetes, $n(\%)$} \\
\hline Yes & $107(14.0)$ & $60(31.6)$ & $47(8.2)$ & $<0.001$ \\
\hline No & $656(86.0)$ & $130(68.4)$ & $526(91.8)$ & - \\
\hline Missing & 3 & 1 & 2 & - \\
\hline \multicolumn{5}{|l|}{$\begin{array}{l}\text { Asthma/chronic obstructive } \\
\text { pulmonary disease, } n(\%)\end{array}$} \\
\hline Yes & $26(3.4)$ & $12(6.3)$ & $14(2.4)$ & 0.011 \\
\hline No & 738 (96.6) & $179(93.7)$ & $559(97.6)$ & - \\
\hline Missing & 2 & 0 & 2 & - \\
\hline \multicolumn{5}{|l|}{ Chronic kidney disease, $n$ (\%) } \\
\hline Yes & $7(0.9)$ & $3(1.6)$ & $4(0.7)$ & 0.375 \\
\hline No & 759 (99.1) & $188(98.4)$ & $571(99.3)$ & - \\
\hline \multicolumn{5}{|l|}{ Cancer, $n(\%)$} \\
\hline Yes & $5(0.6)$ & $3(1.6)$ & $2(0.4)$ & 0.102 \\
\hline No & $761(99.4)$ & $188(98.4)$ & $573(99.6)$ & - \\
\hline \multicolumn{5}{|l|}{$\begin{array}{l}\text { Pregnancy among females, } n \\
(\%)\end{array}$} \\
\hline Yes & $12(4.6)$ & $3(5.4)$ & $9(4.4)$ & 0.720 \\
\hline No & $250(95.4)$ & $52(94.6)$ & $198(95.6)$ & - \\
\hline \multicolumn{5}{|l|}{$\mathrm{SpO}_{2}, n(\%)$} \\
\hline$<90 \%$ & $195(38.2)$ & $166(92.2)$ & $29(8.8)$ & $<0.001$ \\
\hline$\geq 90 \%$ & $315(61.8)$ & $14(7.8)$ & 301 (91.2) & - \\
\hline Missing & 256 & 11 & 245 & - \\
\hline \multicolumn{5}{|l|}{ HIV positive, $n(\%)$} \\
\hline Yes & $12(1.6)$ & $3(1.6)$ & $9(1.6)$ & 1.000 \\
\hline No & $752(98.4)$ & $188(98.4)$ & $564(98.4)$ & - \\
\hline Missing & 2 & 0 & 2 & - \\
\hline \multicolumn{5}{|l|}{ Current tuberculosis, $n(\%)$} \\
\hline Yes & $19(2.5)$ & $4(2.1)$ & $15(2.6)$ & 0.795 \\
\hline No & 745 (97.5) & $187(97.9)$ & $558(97.4)$ & - \\
\hline Missing & 2 & 0 & 2 & - \\
\hline $\mathrm{SpO}_{2}$ (median, IQR), $N$ & $89.0(85-98) 510$ & $79(66-87) 180$ & 98(95-99) 330 & $<0.001$ \\
\hline $\begin{array}{l}\text { Blood glucose (median, IQR) } \\
\text { (mg/dL), } N\end{array}$ & 105 (23-182) 33 & $25(14.5-167.5) 16$ & $131(103-182) 17$ & 0.031 \\
\hline $\begin{array}{l}\text { Serum C-reactive protein } \\
\quad \text { (median, IQR) (mg/dL), } N\end{array}$ & $32(3.3-60) 37$ & $60(48-192) 7$ & $24(2.6-54) 30$ & 0.010 \\
\hline $\begin{array}{l}\text { Serum potassium (median, IQR) } \\
\quad(\mathrm{mEq} / \mathrm{L}), N\end{array}$ & $3.9(3.4-4.3) 17$ & $4.3(2.9-4.8) 3$ & $3.9(3.4-4.0) 14$ & 0.488 \\
\hline $\begin{array}{l}\text { Blood urea nitrogen, median } \\
\text { (mg/dL), } N\end{array}$ & $32.5(21.0-52.0) 46$ & $49.7(41.0-63.0) 14$ & $23.1(20.0-42.2) 32$ & 0.002 \\
\hline Serum creatinine, $(\mathrm{mg} / \mathrm{dL}), N$ & $1.0(0.9-1.2) 48$ & $1.2(1.0-2.0) 13$ & $1.0(0.8-1.1) 35$ & 0.008 \\
\hline $\begin{array}{l}\text { Plasma D-dimer (median, IQR) } \\
\text { (ng/mL), } N\end{array}$ & $183(6.87-349) 11$ & $342.5(246-443) 6$ & $6.9(2.8-100) 5$ & 0.011 \\
\hline \multicolumn{5}{|l|}{ Electrocardiogram, $n(\%)$} \\
\hline Normal & $15(20.6)$ & $1(2.7)$ & $14(38.9)$ & $<0.001$ \\
\hline Abnormal & $58(79.4)$ & $36(97.3)$ & $22(61.1)$ & - \\
\hline Missing & 693 & 154 & 539 & - \\
\hline Chloroquine + azithromycin & $630(86.8)$ & $152(80.8)$ & $478(88.8)$ & 0.005 \\
\hline Other ${ }^{*}$ & 96 (13.2) & 36 (19.2) & 60 (11.2) & - \\
\hline Missing & 24 & 2 & 4 & - \\
\hline
\end{tabular}

* Third-generation cephalosporin and/or amoxicillin +clavulanic acid and/or lopinavir/ritonavir and/or dexamethasone and/or azithromycin. 


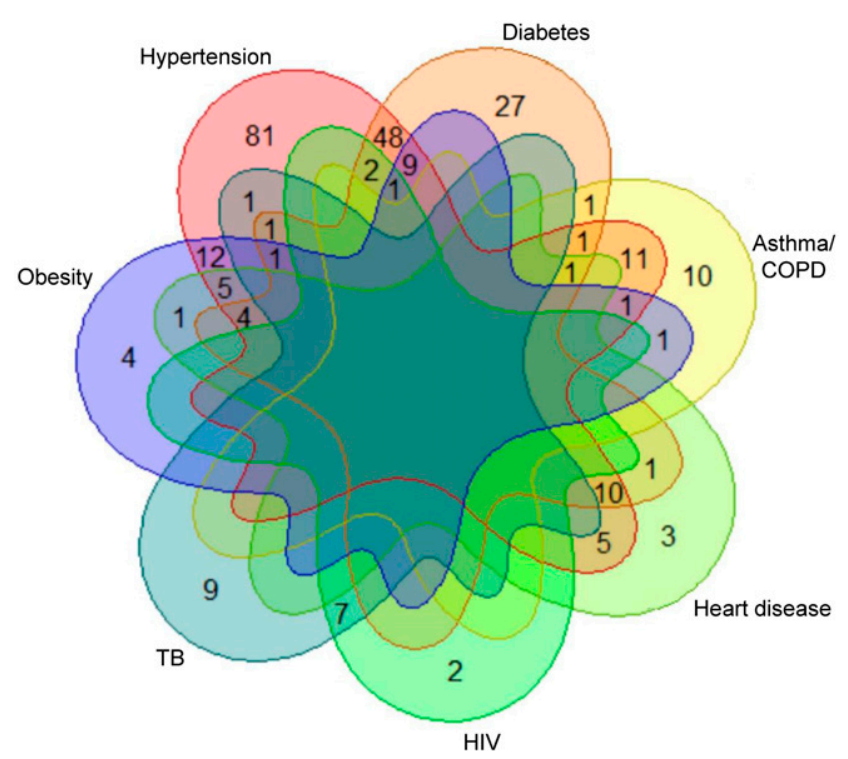

FIGURE 2. Venn diagram showing overlapping between the main comorbidities among COVID-19 hospitalized patients. Patients with chronic kidney disease (CKD) $(n=7)$ and those with cancer $(n=4)$ were not included in the Venn diagram because of the limitation of the package for a maximum of seven comorbidities. Of the seven patients with CKD, three had concomitant hypertension and diabetes $(n=3)$, $\operatorname{DM}(n=3)$, and HTN $(n=1)$. Among the four patients with cancer, one had concomitant heart disease.

more patients with comorbidities died than those without comorbidities. Among the four children who died, one had diabetes and hypertension and the rest had no comorbidities. Mortality among patients with diabetes was greater than nondiabetics (27.1\% versus $10.8 \%$, respectively, $P<0.001)$. More obese versus nonobese patients died (43.6\% versus $11.4 \%, P<0.001)$. The hazard of death among obese patients was more than double than that for nonobese patients $(\mathrm{aHR}=$ 2.30, 95\% Cl: 1.24-4.27). Compared with those without CKD, patients with CKD were at a higher risk of death $(57.1 \%$ versus 12.8, $P<0.001)$, with a more than 5-fold increase in the hazard of death $(\mathrm{aHR}=5.33,95 \% \mathrm{Cl}: 1.85-15.35)$. Patients who received CQ/AZ had significantly lower mortality than those who did not receive these drugs $(11.0 \%$ versus $29.2 \%$, respectively, $P<0.001)$. Mortality in patients receiving supplemental oxygen was greater than that among those who did not (37.6\% versus $2.1 \%$, respectively, $P<0.001$ ). Patients who received $C Q / A Z$ had a $74 \%$ reduction in hazard of death compared with those who did not receive $\mathrm{CQ} / \mathrm{AZ}(\mathrm{aHR}=0.26$, 95\% Cl: 0.16-0.42). However, in MSM analysis, there was no statistically significant difference in risk of death $(\mathrm{aOR}=0.65$, $95 \% \mathrm{Cl}: 0.35-1.20, P=0.166$ ) when comparing use of $\mathrm{CQ} / \mathrm{AZ}$ versus other treatment regimens.

\section{DISCUSSION}

This study is among the first to report clinical characteristics and outcomes of hospitalized COVID-19 patients in an African country. In this hospitalized Congolese cohort, $4.5 \%$ of patients were children $<20$ years, which is similar to studies from China, ${ }^{14}$ Europe, ${ }^{15}$ and the United States ${ }^{16}$ that have reported between $1 \%$ and $5 \%$ of infections in children. Given that SARS-CoV-2 testing is more frequently prompted by symptoms and children typically have asymptomatic or mild infection, the frequency of SARS-CoV-2 infection in Congolese children is likely to be higher than $5 \% .{ }^{17}$ Similar to Asian and Western cohorts, we observed male gender preponderance and previously reported presenting symptoms, including cough, fever, dyspnea, headache, sore throat, and rhinorrhea. ${ }^{18}$ Age and cardiometabolic comorbidities were associated with more severe forms of COVID-19 at admission and a higher risk of death. Unlike other reports, anosmia and dysgeusia were not documented in our cohort. Not surprisingly, patients admitted with severe COVID-19 were more likely to require oxygen therapy; these patients also differed from those with milder COVID-19 in terms of higher levels of inflammatory markers.

In-hospital mortality was $13.2 \%$ in our study population. Global estimates of in-hospital mortality from COVID-19 range between $15 \%$ and $20 \%$, with up to $40 \%$ of hospitalized patients requiring intensive care. ${ }^{18}$ In Western countries, people of African descent and other racial minorities are at increased risk of worse clinical outcomes. ${ }^{19}$ In a recent U.S. cohort, age and proportion of inpatients with comorbidities were higher than our those in the Congolese cohort: mean age: 54 versus 48 years; hypertension: $44 \%$ versus 30\%; diabetes: $39 \%$ versus $16 \%$; obesity: $35 \%$ versus $3.8 \%$; respectively. ${ }^{20}$ Furthermore, our overall in-hospital mortality rate $(13.2 \%)$ may have been influenced by hospitalization of patients with mild disease who may been admitted because of inadequate care and isolation at home due to overcrowding and/or poverty. However, in-hospital mortality was greater among patients with severe/critical disease than among patients those with $\mathrm{mild} /$ moderate disease (45.0\% versus $2.6 \%$, respectively, $P<$ 0.001 ), which is higher than Western reports ${ }^{20}$ but similar to the $\sim 50 \%$ mortality of patients requiring admission to the ICU in a South African cohort. ${ }^{21}$ Of note, dexamethasone has recently been shown to reduce mortality by one-third among seriously ill COVID-19 patients requiring oxygen or respiratory support. The drug was introduced in the DRC's national COVID-19 treatment guidelines ${ }^{12}$ only from July 2020 (last month of our study period), soon after the U.K. Recovery Trial Press Release. ${ }^{22}$ Therefore, further evaluation is needed to ascertain whether in-ICU mortality will decrease with the use of dexamethasone in the DRC.

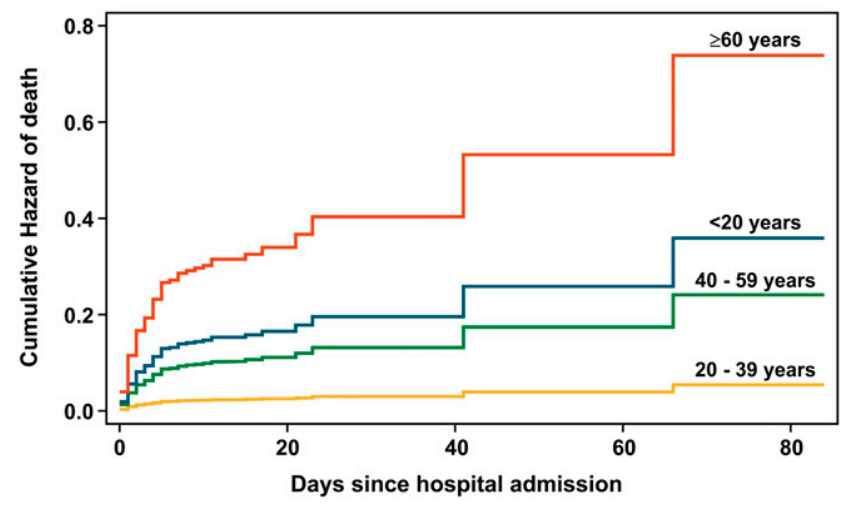

FIGURE 3. Cumulative hazard of death over time stratified by agegroup. The steps in the graph indicate points at which patients died. Patients discharged were censored at time of discharge. The time axis extends to 80 days because that is the longest a patient stayed in hospital. 
TABLE 2

Logistic regression of factors associated with clinical improvement within 30 days $(N=766)$

\begin{tabular}{|c|c|c|c|c|}
\hline Characteristic & Improved, $n(\%)$ & Unadjusted odds ratio $(95 \% \mathrm{Cl})$ & Adjusted odds ratio (95\% Cl) & $P$-value \\
\hline \multicolumn{5}{|l|}{ Gender } \\
\hline Female $(n=262)$ & $211(80.5)$ & 1 & - & - \\
\hline Male $(n=500)$ & 406 (81.2) & $1.04(0.71-1.52)$ & - & - \\
\hline \multicolumn{5}{|l|}{ Age-group (years) } \\
\hline$<20(n=34)$ & $29(85.3)$ & $3.58(1.32-9.71)$ & $2.98(1.05-8.49)$ & 0.041 \\
\hline $20-39(n=248)$ & $233(94.0)$ & $9.60(5.25-17.55)$ & $9.40(4.77-18.52)$ & $<0.001$ \\
\hline $40-59(n=303)$ & 246 (81.9) & $2.67(1.76-4.05)$ & $2.64(1.64-4.26)$ & $<0.001$ \\
\hline$\geq 60(n=178)$ & $110(61.8)$ & 1 & 1 & - \\
\hline \multicolumn{5}{|l|}{ Clinical stage at presentation } \\
\hline Mild or moderate $(n=575)$ & $525(91.3)$ & 1 & - & - \\
\hline Severe or critical $(n=191)$ & $95(49.7)$ & $0.09(0.06-0.14)$ & - & - \\
\hline \multicolumn{5}{|l|}{ Hypertension } \\
\hline No $(n=570)$ & $480(84.2)$ & 1 & 1 & - \\
\hline Yes $(n=194)$ & $139(71.6)$ & $0.47(0.32-0.70)$ & $1.28(0.76-2.18)$ & 0.356 \\
\hline \multicolumn{5}{|l|}{ Heart disease } \\
\hline No $(n=733)$ & $600(81.9)$ & 1 & 1 & - \\
\hline Yes $(n=30)$ & $18(60.0)$ & $0.33(0.16-0.71)$ & $0.81(0.32-2.03)$ & 0.656 \\
\hline \multicolumn{5}{|l|}{ Diabetes } \\
\hline No $(n=656)$ & $547(83.4)$ & 1 & 1 & - \\
\hline Yes $(n=107)$ & $71(66.4)$ & $0.39(0.25-0.62)$ & $0.76(0.43-1.35)$ & 0.351 \\
\hline \multicolumn{5}{|l|}{ Obesity } \\
\hline No $(n=725)$ & $600(82.8)$ & 1 & 1 & - \\
\hline Yes $(n=39)$ & $19(48.7)$ & $0.20(0.10-0.38)$ & $0.27(0.12-0.59)$ & 0.001 \\
\hline \multicolumn{5}{|c|}{$\begin{array}{l}\text { Asthma/chronic obstructive pulmonary } \\
\text { disease }\end{array}$} \\
\hline No $(n=738)$ & $600(81.3)$ & 1 & - & - \\
\hline Yes $(n=26)$ & 19 (73.1) & $0.62(0.26-1.51)$ & - & - \\
\hline \multicolumn{5}{|l|}{ Chronic kidney disease } \\
\hline No $(n=759)$ & $617(81.3)$ & 1 & 1 & - \\
\hline Yes $(n=7)$ & 3 (42.9) & $0.17(0.04-0.78)$ & $0.22(0.04-1.08)$ & 0.063 \\
\hline \multicolumn{5}{|l|}{ Cancer } \\
\hline No $(n=761)$ & 618 (81.2) & 1 & 1 & - \\
\hline Yes $(n=5)$ & $2(40)$ & $0.15(0.02-0.93)$ & $0.38(0.06-2.50)$ & 0.313 \\
\hline \multicolumn{5}{|l|}{ HIV } \\
\hline No $(n=752)$ & $611(81.2)$ & 1 & - & - \\
\hline Yes $(n=12)$ & 8 (66.7) & $0.46(0.14-1.55)$ & - & - \\
\hline \multicolumn{5}{|l|}{ Current tuberculosis } \\
\hline No $(n=745)$ & 604 (81.1) & 1 & - & - \\
\hline Yes $(n=19)$ & $15(79.0)$ & $0.88(0.29-2.68)$ & - & - \\
\hline \multicolumn{5}{|c|}{$\begin{array}{l}\text { Chloroquine/azithromycin-based } \\
\text { treatment vs. other }\end{array}$} \\
\hline No $(n=96)$ & $62(64.6)$ & 1 & 1 & - \\
\hline Yes $(n=630)$ & $526(83.5)$ & $2.77(1.74-4.43)$ & $3.62(2.12-6.16)$ & $<0.001$ \\
\hline \multicolumn{5}{|l|}{ Received oxygen } \\
\hline No $(n=330)$ & 307 (93.0) & 1 & - & - \\
\hline Yes $(n=245)$ & 137 (55.9) & $0.10(0.06-0.16)$ & - & - \\
\hline
\end{tabular}

Among the comorbidities evaluated, hypertension and diabetes were clearly associated with more severe presentation and poorer prognosis for COVID-19. This is in line with findings published from China, the United States, and Europe. ${ }^{14-16}$ These two comorbidities were strongly co-prevalent in our cohort, with $38 \%$ of hypertensive patients being diabetic, and $70 \%$ of diabetics being hypertensive. More importantly, despite the overall prevalence of self-reported obesity being low (potentially conservative bias due to underestimation), obesity was a significant independent predictor of mortality. Early studies suggest that cytokine release is central to the development of COVID-19-related respiratory distress, ${ }^{20}$ that interleukin-6 (IL-6) is produced by multiple cells including adipocytes, ${ }^{23,24}$ and that IL-6 levels are elevated in obese individuals. ${ }^{25,26}$ Furthermore, adipose tissue has been hypothesized to be a site for SARS-CoV-2 replication and shedding. ${ }^{27}$

In the French Coronavirus SARS-CoV-2 and Diabetes Outcomes (CORONADO) study, among diabetic inpatients with COVID-19, body mass index and poor long-term glucose control were independently associated with mechanical ventilation and/or death. ${ }^{28}$ Several arguments suggest that there is no causal link between severe pneumonia and chronic hyperglycemia and that the overrepresentation of diabetic patients with COVID-19 in ICUs indirectly reflects the impact of obesity. ${ }^{29}$ Furthermore, higher hemoglobin A1c (HbA1c) at admission does not appear to worsen COVID-19 prognosis in type II diabetes. ${ }^{30}$ For this study, there were no $\mathrm{HbA} 1 \mathrm{c}$ data available; thus, we were unable to analyze its potential association with COVID-19 outcomes. We also found that CKD was an independent risk factor for mortality, as reported from outside Africa. ${ }^{31-34}$ Patients presenting with SARS-CoV-2 infection have shown varying degrees of renal dysfunction, including a high incidence of acute kidney injury. ${ }^{32,35}$ A recent study reported that the human kidney may be a unique target for SARS-CoV-2 because it expresses angiotensin-converting enzyme-2 surface receptors. ${ }^{32,33,35}$

There was no significant difference in mortality when comparing $\mathrm{CQ} / \mathrm{AZ}$ versus other regimens by MSM analysis. Our 
TABLE 3

Cox regression of factors associated with hazard of death $(N=766)$

\begin{tabular}{|c|c|c|c|c|}
\hline Characteristic & Died, $n(\%)$ & Unadjusted hazards ratio $(95 \% \mathrm{Cl})$ & Adjusted hazards ratio $(95 \% \mathrm{Cl})^{*}$ & $P$-value \\
\hline \multicolumn{5}{|l|}{ Gender } \\
\hline Female $(n=262)$ & $34(13.0)$ & 1 & - & - \\
\hline Male $(n=500)$ & 67 (13.4) & $1.03(0.68-1.56)$ & - & - \\
\hline \multicolumn{5}{|l|}{ Age-group (years) } \\
\hline$<20(n=34)$ & $4(11.8)$ & $5.10(1.44-18.09)$ & $6.62(1.85-23.65)$ & 0.004 \\
\hline $20-39(n=248)$ & $6(2.4)$ & 1 & 1 & - \\
\hline $40-59(n=303)$ & $34(11.2)$ & $4.62(1.94-11.01)$ & $4.45(1.83-10.79)$ & 0.001 \\
\hline$\geq 60(n=178)$ & 57 (32.0) & $14.85(6.40-34.46)$ & $13.63(5.70-32.60)$ & $<0.001$ \\
\hline \multicolumn{5}{|l|}{ Clinical stage at admission } \\
\hline Mild or moderate $(n=575)$ & $15(2.6)$ & 1 & - & - \\
\hline Severe or critical $(n=191)$ & $86(45.0)$ & $20.84(12.02-36.14)$ & - & - \\
\hline \multicolumn{5}{|l|}{ Hypertension } \\
\hline No $(n=570)$ & $56(9.8)$ & 1 & 1 & - \\
\hline Yes $(n=194)$ & $44(22.7)$ & $2.32(1.56-3.45)$ & $1.00(0.62-1.61)$ & 0.986 \\
\hline \multicolumn{5}{|l|}{ Heart disease } \\
\hline No $(n=733)$ & $89(12.1)$ & 1 & 1 & - \\
\hline Yes $(n=30)$ & $11(36.7)$ & $3.52(1.88-6.60)$ & $1.40(0.68-2.88)$ & 0.364 \\
\hline \multicolumn{5}{|l|}{ Diabetes } \\
\hline No $(n=656)$ & $71(10.8)$ & 1 & 1 & - \\
\hline Yes $(n=107)$ & $29(27.1)$ & $2.53(1.64-3.91)$ & $1.10(0.66-1.81)$ & 0.720 \\
\hline \multicolumn{5}{|l|}{ Obesity } \\
\hline No $(n=725)$ & $83(11.4)$ & 1 & 1 & - \\
\hline Yes $(n=39)$ & $17(43.6)$ & $3.87(2.86-6.56)$ & $2.30(1.24-4.27)$ & 0.009 \\
\hline \multirow{2}{*}{\multicolumn{5}{|c|}{$\begin{array}{l}\text { Asthma/chronic obstructive pulmonary } \\
\text { disease }\end{array}$}} \\
\hline & & & & \\
\hline No $(n=738)$ & $96(13.0)$ & 1 & - & - \\
\hline Yes $(n=26)$ & $4(15.4)$ & $1.27(0.46-3.45)$ & - & - \\
\hline \multicolumn{5}{|l|}{ Chronic kidney disease } \\
\hline No $(n=759)$ & $97(12.8)$ & 1 & 1 & - \\
\hline Yes $(n=7)$ & $4(57.1)$ & $5.33(1.96-14.52)$ & $5.33(1.85-15.35)$ & 0.002 \\
\hline \multicolumn{5}{|l|}{ Cancer } \\
\hline No $(n=761)$ & $99(13.0)$ & 1 & - & \\
\hline Yes $(n=5)$ & $2(40.0)$ & $3.90(0.96-15.82)$ & - & - \\
\hline \multicolumn{5}{|l|}{ HIV } \\
\hline No $(n=752)$ & $98(13.0)$ & 1 & - & - \\
\hline Yes $(n=12)$ & $2(16.7)$ & $1.23(0.30-4.99)$ & - & - \\
\hline \multicolumn{5}{|l|}{ Current tuberculosis } \\
\hline No $(n=745)$ & $98(13.2)$ & 1 & - & - \\
\hline Yes $(n=19)$ & $2(10.5)$ & $0.73(0.18-2.98)$ & - & - \\
\hline \multicolumn{5}{|c|}{$\begin{array}{l}\text { Chloroquine/azithromycin-based } \\
\text { treatment }\end{array}$} \\
\hline No $(n=96)$ & $28(29.2)$ & 1 & 1 & - \\
\hline Yes $(n=630)$ & $69(11.0)$ & $0.33(0.21-0.52)$ & $0.26(0.16-0.42)$ & $<0.001$ \\
\hline \multicolumn{5}{|l|}{ Received oxygen } \\
\hline No $(n=330)$ & $7(2.1)$ & 1 & - & - \\
\hline Yes $(n=245)$ & $92(37.6)$ & $21.88(10.14-47.25)$ & - & - \\
\hline
\end{tabular}

data do not conclusively exclude a $C Q / A Z$ treatment effect, given the lack of a comparator arm, and nonrandom treatment allocation. However, recently published placebo-controlled trials from the United States, ${ }^{36}$ the United Kingdom, ${ }^{37}$ and Brazil $^{38}$ have shown no effect of CQ or CQ/AZ on COVID-19 mortality. There is an urgent need for rigorous evaluation of other promising and scalable cost-effective therapeutic options for the DRC and other African countries.

Our findings showed no association of HIV and/or TB with baseline COVID-19 disease severity or prognosis. However, definitive conclusions cannot be made because of low prevalence of HIV (1.6\%) and active TB (2.2\%) in our cohort. A population-based U.K. study found that HIV-positive individuals had more than double the risk of COVID-19-related mortality than HIV-uninfected individuals after controlling for known confounding factors. ${ }^{39}$ Similarly, a retrospective analysis of $>20,000$ South African adults with COVID-19 showed that HIV was associated with a doubling of COVID-19 mortality risk, although this may be an overestimate because of residual confounders. ${ }^{40}$
Larger SSA cohort studies are required to further define the epidemiological, clinical, and risk relationships among the overlapping epidemics of COVID-19, HIV, TB, and malaria.

Of great concern is that children and adolescents $<20$ years had a CFR of $11.8 \%$ and were nearly seven times more likely to die than patients aged 20-39 years. By contrast, studies mostly from China report CFRs of $<1 \%$ among both symptomatic and asymptomatic, but mostly hospitalized children. ${ }^{41,42}$ A recent U.S. study reported a CFR of $2 \%$ among children hospitalized with COVID-19). ${ }^{43}$ All four pediatric deaths in our DRC cohort occurred among older children aged 16-19 years. Three of the four had severe/critical disease, and one had moderate disease at admission; also, three of these four cases had no underlying comorbidity. Webb et al. ${ }^{44}$ recently reported on 23 South African children with MIS-C, among whom $52 \%$ required ICU admission primarily because of cardiac dysfunction. There were no deaths reported in this South African cohort; all the children survived. Multisystem inflammatory syndrome was not specifically 
reported in our DRC pediatric cohort, but it may not have been recognized. Our study's small number of children and the possibility of unmeasured confounding factors, such as the availability of necessary equipment, quality, and scope of pediatric intensive care, preclude concrete conclusions about excess COVID-19-related mortality among children and adolescents in the DRC. This calls for larger, robust investigations of COVID-19 outcomes among hospitalized children in SSA.

Our study has some limitations. Approximately $10 \%$ of patients had missing data on outcomes of interest and were not included in our analysis. However, these patients were comparable with those included with respect to sociodemographic characteristics and COVID-19 clinical stage. In addition, we were not able to compare clinical characteristics between hospitalized COVID-19 patients and outpatients. Finally, given the low prevalence of self-reported HIV and/or TB status, we cannot speculate on the impact of these conditions on COVID-19 outcomes. Strengths of our study include a robust sample size of hospitalized patients in SSA from where little information on COVID-19 has been reported. We also provide data on 34 children, a population for whom there are even less COVID-19 data available from SSA. ${ }^{9} \mathrm{Fi}-$ nally, the use of robust statistical methods such as MSM and IPTW creates more balanced comparisons between treatment groups, similar to those that would be found in a randomized clinical trial.

\section{CONCLUSION}

In this study, hospitalized patients in SSA with COVID-19 had a somewhat lower overall in-hospital mortality than hospitalized patients in non-African regions, but mortality in those with severe or critical disease was almost 50\%. Age-groups at high risk and comorbidities associated with death were similar between our cohort and those from prior studies in Asia, Europe, and North America. Although our study provides insights into COVID-19 manifestations in Africa, more data are needed from countries across this region. Large-cohort observational studies are required to better define the epidemiology and factors affecting COVID-19 outcomes and the relationships between the overlapping epidemics of COVID-19, HIV, TB, and malaria among both young and older populations. Rigorous evaluations of promising, scalable, and cost-effective therapeutics and vaccines are needed globally.

Received September 21, 2020. Accepted for publication September 27, 2020.

Published online October 2, 2020.

Note: Supplemental tables appear at www.ajtmh.org.

Acknowledgments: We acknowledge critical review by John L. Johnson, Case Western Reserve University, Cleveland, OH; Todd Brown, Johns Hopkins University, Baltimore, MD; Michael Reid, University of California, San Francisco, CA; Christina Wyatt, Durham, NC; Peter Kilmarx, NIH/FIC, Bethesda, MD; Wolfgang Preiser and Eric Decloedt, both from Stellenbosch University, Cape Town, South Africa; Andre-Pascal Kengne, South African Medical Research Council (SAMRC), Cape Town, South Africa; and Rodney Ehrlich, and Mary-Ann Davies, University of Cape Town, Cape Town, South Africa. Publication charges for this article were waived due to the ongoing pandemic of COVID-19.

Financial support: J. B. N. is an infectious diseases internist and epidemiologist supported by the U.S. NIH/National Institutes of Allergy and Infectious Diseases grant number 5U01AI069521 (Stellenbosch University Clinical Trial Unit of the AIDS Clinical Trial Group) as well as $\mathrm{NIH} /$ Fogarty International Center grant numbers 1R25TW011217-01 (African Association for Health Professions Education and Research) and 1D43TW010937-01A1 (University of Pittsburgh HIV Comorbidities Research Training Program in South Africa) and is coprincipal investigator of TOGETHER, an adaptive randomized clinical trial of novel agents for treatment of high-risk outpatient COVID-19 patients in South Africa supported by the Bill \& Melinda Gates Foundation. DKI, CB-PN, PM-K, ENK, GMM, JMS, MTP, SA-M, J-JMT, DJML, and JMK are members of the DRC Ministry of Health's COVID-19 MultiSectoral Response Committee. A. Z. is a coprincipal investigator of the Pan-African Network on Emerging and Re-Emerging Infections (PANDORA-ID-NET; https://www.pandora-id.net/) funded by the EU Horizon 2020 Framework Program for Research and Innovation and is in receipt of an U.K. NIH Research Senior Investigator award. N. A. S. A. is a clinician-scientist with pediatric infectious disease expertise and is funded by the $\mathrm{NIH} / \mathrm{National}$ Institute of Child Health and Human Development grant R01HD089866, and by an NIH/Fogarty International Center award under the Adolescent HIV Prevention and Treatment Implementation Science Alliance (AHISA), for the Central and West Africa Implementation Science Alliance (CAWISA).

Authors' addresses: Jean B. Nachega, Department of Medicine, Centre for Infectious Diseases, Faculty of Medicine and Health Sciences, Stellenbosch University, Cape Town, South Africa, Department of Epidemiology and International Health, Johns Hopkins Bloomberg School of Public Health, Baltimore, MD, and Department of Epidemiology, Infectious Diseases and Microbiology, and Center for Global Health, University of Pittsburgh, Pittsburgh, PA, E-mail: jbn16@pitt.edu. Daniel Katuashi Ishoso, Community Health Department, Kinshasa School of Public Health, University of Kinshasa, Kinshasa, Democratic Republic of the Congo, E-mail: dishosok@ gmail.com. John Otshudiema Otokoye, Joule Ntwan Madinga, and Marie Claire Kolié, Epidemiological Surveillance Team, COVID-19 Response, Health Emergencies Program, World Health Organization, Kinshasa, Democratic Republic of the Congo, E-mails: johnotokoye@ gmail.com, jmadinga@yahoo.fr, and marieclairekolie@gmail.com. Michel P. Hermans, Department of Endocrinology and Nutrition, Cliniques Universitaires St-Luc, Brussels, Belgium, E-mail:michel.hermans@ uclouvain.be. Rhoderick Neri Machekano, African Center of Biostatistics Excellence (ACBE), Faculty of Medicine and Health Sciences, Stellenbosch University, Cape Town, South Africa, E-mail: rhoderick@ sun.ac.za. Nadia A. Sam-Agudu, Institute of Human Virology Nigeria, International Research Center of Excellence, Abuja, Nigeria, and Division of Epidemiology and Prevention, Institute of Human Virology, Baltimore, MD, E-mail: nsamagudu@ihvnigeria.org. Christian Bongo-Pasi Nswe and Don Jethro Mavungu Landu, Department of Public Health, Centre Interdisciplinaire de Recherche en Ethnopharmacologie, Faculty of Medicine, Université Notre-Dame du Kasayi, Kananga, Democratic Republic of the Congo, and Faculty of Public Health, Université Moderne de Kinkole, Kinshasa, Democratic Republic of the Congo, E-mails: bongopanoudji@gmail.com and jethromavungu@gmail.com. Placide Mbala-Kingebeni, Department of Microbiology, Institut National de Recherche Biomedicale, Kinshasa, Democratic Republic of the Congo, E-mail: mbalaplacide@gmail.com. Stéphane Mukendi, Edith N. Nkwembe, and Jean-Jacques MuyembeTamfum, Department of Virology, Faculty of Medicine, National Institute of Biomedical Research (INRB), University of Kinshasa, Kinshasa, Democratic Republic of the Congo, E-mails: mukendisteph1@gmail.com, edithnkwembe1@gmail.com, and jjmuyembet@gmail.com. Steve Ahuka-Mundeke, Technical Secretariat, Ebola Response, Goma, Democratic Republic of the Congo, E-mail: amstev04@yahoo.fr. Gisele M. Mbuyi and Justus M. Nsio, Direction Surveillance Épidémiologique (DSE), Direction Générale de Lutte contre la Maladie (DGLM), Ministère de la Santé Publique et Riposte COVID-19, Kinshasa, Democratic Republic of the Congo, E-mails: drgmbuyi@ gmail.com and justsusnsio@gmail.com. Didier Mukeba Tshialala, Department of Medicine, Faculty of Medicine, University of MbujiMayi, Mbuji-Mayi, Democratic Republic of the Congo, E-mail: dimutshia@yahoo.fr. Michel Tshiasuma Pipo, Department of Public Health, Faculty of Medicine, Centre Interdisciplinaire de Recherche en Ethnopharmacologie, Université Notre-Dame du Kasayi, Kananga, Democratic Republic of the Congo, E-mail: mycky1974@gmail.com. Lynne Mofenson, Elizabeth Glaser Pediatric AIDS Foundation, Washington, DC, E-mail: mofensol@gmail.com. Gerald Smith, 
Department of Real World \& Advanced Analytics, Cytel, Vancouver, Canada, E-mail: gerald.smith@cytel.com. Edward J. Mills, Department of Health Research Methods, Evidence, and Impact, McMaster University, Hamilton, Canada, E-mail: emills@mteksciences.com. John W. Mellors, Division of Infectious Diseases, Department of Medicine, University of Pittsburgh, School of Medicine, Pittsburgh, PA, E-mail: jwm1@pitt.edu. Alimuddin Zumla, Division of Infection and Immunity, Department of Infection, University College London, London, United Kingdom, E-mail: a.zumla@ucl.ac.uk. Jean-Marie Kayembe, Department of Medical Microbiology and Virology, Faculty of Medicine, National Institute of Biomedical Research (INRB), University of Kinshasa, Kinshasa, Democratic Republic of the Congo, E-mail: jm.kayembe@unikin.ac.cd.

This is an open-access article distributed under the terms of the Creative Commons Attribution (CC-BY) License, which permits unrestricted use, distribution, and reproduction in any medium, provided the original author and source are credited.

\section{REFERENCES}

1. World Health Organization, 2020. COVID-19 Dashboard. Available at: https://covid19.who.int/. Accessed September 20, 2020.

2. World Health Organization, 2020. New WHO Estimates: Up to 190 000 People Could Die of COVID-19 in Africa if Not Controlled. Available at: https://www.afro.who.int/news/new-who-estimates190-000-people-could-die-covid-19-africa-if-not-controlled. Accessed August 24, 2020.

3. Nachega JB, Seydi M, Zumla A, 2020. The late arrival of COVID-19 in Africa-mitigating pan-continental spread. Clin Infect Dis 71: 875-878.

4. Mehtar S, Preiser W, Lakhe NA, Bousso A, TamFum JM, Kallay O, Seydi M, Zumla A, Nachega JB, 2020. Limiting the spread of COVID-19 in Africa: one size mitigation strategies do not fit all countries. Lancet Glob Health 8: e881-e883.

5. Ayoub HH, Chemaltelly H, Seedat S, Mumtaz GR, Makhoul M, AbuRaddad LJ, 2020. Age could be driving variable SARS-CoV-2 epidemic trajectories worldwide. PLoS One 15: e0237959.

6. Nachega JB et al., 2020. From easing lockdowns to scaling-up community-based COVID-19 screening, testing, and contact tracing in Africa-shared approaches, innovations, and challenges to minimize morbidity and mortality. Clin Infect Dis ciaa695. doi: 10.1093/cid/ciaa695.

7. Hale T, Webster S, Petherick A, Phillips T, Kira B, 2020. Oxford COVID-19 Government Response Tracker, Blavatnik School of Government. Available at: https://www.bsg.ox.ac.uk/research/ research-projects/coronavirus-government-response-tracker. Accessed August 24, 2020.

8. Massinga Loembé M, Tshangela A, Salyer SJ, Varma JK, Ouma AEO, Nkengasong JN, 2020. COVID-19 in Africa: the spread and response. Nat Med 26: 999-1003.

9. Coker M, Folayan MO, Michelow IC, Oladokun RE, Torbunde N, Sam-Agudu NA, 2020. Things must not fall apart: the ripple effects of the COVID-19 pandemic on children in sub-saharan Africa. Pediatr Res (Epub ahead of print). doi: 10.1038/s41390020-01174-y.

10. Amimo F, Lambert B, Magit A, 2020. What does the COVID-19 pandemic mean for HIV, tuberculosis, and malaria control? Trop Med Health 48: 32

11. World Health Organization, 2020. Clinical Management of COVID-19. Available at: https://www.who.int/publications/i/ item/clinical-management-of-covid-19. Accessed August 16, 2020.

12. Democratic Republic of the Congo, Ministry of Health Secretary, 2020. COVID-19 National Case Management Protocol. Protocole de prise en Charge de l'épidémie a Coronavirus COVID-19. Kinshasa, Democratic Republic of Congo: Ministry of Public Health.

13. Chen H, Boutros PC, 2011. Venn diagram: a package for the generation of highly customizable Venn and Euler diagrams in R. BMC Bioinformatics 12: 35.

14. Guan WJ et al., 2020. Clinical characteristics of coronavirus disease 2019 in China. N Engl J Med 382: 1708-1720.
15. Lescure FX et al., 2020. Clinical and virological data of the first cases of COVID-19 in Europe: a case series. Lancet Infect Dis 20: 697-706.

16. Goyal $P$ et al., 2020. Clinical characteristics of COVID-19 in New York city. N Engl J Med 382: 2372-2374.

17. Wald ER, Schmit KM, Gusland DY, 2020. A pediatric infectious disease perspective on COVID-19. Clin Infect Dis ciaa1095. doi: 10.1093/cid/ciaa1095.

18. Wiersinga WJ, Rhodes A, Cheng AC, Peacock SJ, Prescott HC, 2020. Pathophysiology, transmission, diagnosis, and treatment of coronavirus disease 2019 (COVID-19): a review. JAMA 324: 782-793.

19. Pan D et al., 2020. The impact of ethnicity on clinical outcomes in COVID-19: a systematic review. EClinicalMedicine 23: 100404.

20. Joseph NP et al., 2020. Racial/ethnic disparities in disease severity on admission chest radiographs among patients admitted with confirmed COVID-19: a retrospective cohort study. Radiology 202602. doi: 10.1148/radiol.2020202602.

21. Parker A et al., 2020. High HIV prevalence in an early cohort of hospital admissions with COVID-19 in Cape Town, South Africa. S Afr Med J (Epub ahead of print). Available at: http:// www.samj.org.za/index.php/samj/article/view/13054/9454.

22. Horby P, Landrain M; RECOVERY Trial Press Release, 2020. LowCost Dexamethasone Reduces Death by Up to One Third in Hospitalised Patients with Severe Respiratory Complications of COVID-19. Available at: https://www.ox.ac.uk/news/2020-0616-low-cost-dexamethasone-reduces-death-one-third-hospitalised-patients-severe. Accessed July 5, 2020.

23. Liu B, Li M, Zhou Z, Guan X, Xiang Y, 2020. Can we use interleukin-6 blockade for coronavirus disease 2019 (COVID-19)-induced cytokine release syndrome (CRS)? J Autoimmun 111: 102452.

24. Zhou F et al., 2020. Clinical course and risk factors for mortality of adult inpatients with COVID-19 in Wuhan, China: a retrospective cohort study. Lancet 395: 1054-1062.

25. Huang $C$ et al., 2020. Clinical features of patients infected with 2019 novel coronavirus in Wuhan, China. Lancet 395: 497-506.

26. Kahn SE, Hull RL, Utzschneider KM, 2006. Mechanisms linking obesity to insulin resistance and type 2 diabetes. Nature 444: 840-846.

27. Ryan PM, Caplice NM, 2020. Is adipose tissue a reservoir for viral spread, immune activation and cytokine amplification in COVID-19. Obesity 28: 1191-1194.

28. Cariou B et al., 2020. Phenotypic characteristics and prognosis of inpatients with COVID-19 and diabetes: the CORONADO study. Diabetologia 63: 1500-1515.

29. Orioli L, Hermans MP, Thissen JP, Maiter D, Vandeleene B, Yombi JC, 2020. COVID-19 in diabetic patients: related risks and specifics of management. Ann Endocrinol (Paris) 81: 101-109.

30. Zhang YM, Zhang H, 2020. Genetic roadmap for kidney involvement of severe acute respiratory syndrome coronavirus 2 (SARS-CoV-2) infection. Clin J Am Soc Nephrol 15: 1044-1046.

31. Su $\mathrm{H}$ et al., 2020. Renal histopathological analysis of 26 postmortem findings of patients with COVID-19 in China. Kidney Int 98: 219-227.

32. Diao B et al., 2020. Human kidney is a target for novel severe acute respiratory syndrome coronavirus 2 (SARS-CoV-2) infection. medRxiv. Available at: https://doi.org/10.1101/2020.03.04.20031120.

33. Kissling S, Rotman S, Gerber C, Halfon M, Lamoth F, Comte D, Lhopitallier L, Sadallah S, Fakhouri F, 2020. Collapsing glomerulopathy in a COVID-19 patient. Kidney Int 98: 228-231.

34. Alberici $F$ et al., 2020. Management of patients on dialysis and with kidney transplant during SARS-CoV-2 (COVID-19) pandemic in Brescia, Italy. Kidney Int Rep 5: 580-585.

35. Hirsch JS et al.; Northwell COVID-19 Research Consortium, Northwell Nephrology COVID-19 Research Consortium, 2020. Acute kidney injury in patients hospitalized with COVID-19. Kidney Int 98: 209-218.

36. Skipper CP et al., 2020. Hydroxychloroquine in nonhospitalized adults with early COVID-19: a randomized trial. Ann Intern Med M20-M4207. doi: 10.7326/M20-4207.

37. Horby P, Landray M, 2020. No Clinical Benefit from Use of Hydroxychloroquine in Hospitalised Patients with COVID-19. Press Release. Available at: https://www.recoverytrial.net/news/statementfrom-the-chief-investigators-of-therandomised-evaluationof-covid-19-therapy-recovery-trialon-hydroxychloroquine5-june-2020-no-clinical-benefitfrom-use-of-hydroxychloroquinein-hospitalised-patients-withcovid-19. Accessed July 5, 2020. 
38. Cavalcanti $A B$ et al., 2020. Hydroxychloroquine with or without azithromycin in mild-to-moderate COVID-19. N Engl J Med (Epub ahead of print, 2020 July 23) doi: 10.1056/ NEJMoa2019014.

39. Williamson E et al., 2020. OpenSAFELY: Factors Associated with COVID-19-related Hospital Death in the Linked Electronic Health Records of 17 Million Adult NHS Patients. Available at: https://www.medrxiv.org/content/10.1101/2020.05.06.20092999v1. Accessed August 15, 2020.

40. Boulle A. et al., 2020. HIV and risk of COVID-19 death: a population cohort study from the western Cape province, South Africa. Clin Infect Dis ciaa1198. Available at: https://www.medrxiv.org/ content/10.1101/2020.07.02.20145185v2.
41. Castagnoli R, Votto M, Licari A, Brambilla I, Bruno R, Perlini S, Rovida F, Baldanti F, Marseglia GL, 2020. Severe acute respiratory syndrome coronavirus 2 (SARS-CoV-2) infection in children and adolescents: a systematic review. JAMA Pediatr (Epub ahead of print). doi: 10.1001/jamapediatrics.2020.1467.

42. Feldstein LR et al., 2020. Multisystem inflammatory syndrome in U.S. children and adolescents. N Engl J Med 383: 334-346.

43. Mehta NS et al., 2020. SARS-CoV-2 (COVID-19): what do we know about children? A systematic review. Clin Infect Dis ciaa556. doi: 10.1093/cid/ciaa556.

44. Webb K, Abraham DR, Faleye A, McCulloch M, Rabie H, Scott C; Cape Town MISC-Team, 2020. Multisystem inflammatory syndrome in children in South Africa. Lancet Child Adolesc Health 4: e38. 